\title{
ОЦЕНКА ПОКАЗАТЕЛЕЙ РИСКА ПРОИЗВОДСТВЕННОГО ПРЕДПРИЯТИЯ В УСЛОВИЯХ НЕОПРЕДЕЛЕННОСТИ
}

\author{
(C) 2021 Козина Е. В. \\ кандидат экономических наук, доцент кафедры экономика и управление \\ Пензенский государственный технологический университет, Россия, Пенза \\ E-mail: uhelena@list.ru
}

На современном этапе хозяйствования большинство управленческих решений принимаются в условиях риска и неопределенности, это обусловлено рядом причин, а именно: неполнота или неточность знаний об экономическом явлении, наличие противоборствующих тенденций, воздействие элементов случайности и т.д. Поэтому предприятия постоянно сталкиваются с необходимостью оценки и анализа риска, а так же выработки эффективных методов управления (минимизации) риска.

В статье рассмотрена количественная оценка рисков, связанных с альтернативными направлениями деятельности производственного предприятия в условиях неопределенности.

Ключевые слова: риск, оценка, предприятие, производство, анализ, управление.

Предпринимательская деятельность всегда сопряжена с рисками и неопределенностью.

Нынешние условия глобализации и интенсивного инновационного развития еще в большей степени усиливают фактор неопределенности и требуют от современного бизнеса больших усилий по управлению рисками.

Руководство современных предприятий вынуждено прорабатывать новые стратегии, которые в обязательном порядке должны включать оценку и анализ возможных рисков, а так же сценарии развития событий при их возникновении.

В связи с этим растет необходимость в постоянном мониторинге текущей ситуации, проработке методов и средств идентификации и предупреждения рисков, выборе действенных методов по их минимизации.

Риск - опасность возникновения непредвиденных потерь ожидаемой прибыли, дохода или имущества, денежных средств в связи со случайным изменением условий экономической деятельности, неблагоприятными обстоятельствами [5].

Основу риска составляет неопределенность. Неопределенность развития событий и их исхода связана со множеством причин, которые, в свою очередь и являются причинами возникновения риска. Чаще всего в научной литературе выделяют три ключевых причины неопределенности: незнание, случайность и противодействие.

Незнание при этом означает неполноту знаний (отсутствие) о среде функционирования. Случайность представляет собой невозможность абсолютного предвидения исхода ситуации, причем схожесть ситуации и условий не дает гарантии получения одинакового результата. Противодействие здесь означает явления (события), оказывающие тормозящее действие на процесс деятельности предприятия (например, конфликты внутри компании или с контрагентами).

В основе проведения анализа рисков лежит изучение возможностей их проявления и их оценка с целью последующей минимизации и определения возможностей управления.

По своей сути обнаружение рисков и причин их возникновения заключается в анализе степени вероятности возникновения отрицательных ситуаций и прогноза возможных негативных последствий данных ситуаций.

Существует два базовых подхода к анализу рисков: качественный и количественный. Качественный подход заключается в идентификации самих рисков и определении факторов их проявления. Количественный анализ позволяет выразить риски численно за счет использования математических и статистических методов.

С развитием экономической и управленческой наук изменились и методы, используемые в риск-менеджменте. И сегодня эксперты чаще всего при анализе рисков используют комплексный подход.

Именно комплексный подход позволяет получить наиболее полное представление о возможных рисках, оценить вероятные исходы развития ситуации, а также реализовать наибольшее число математических и статистиче- 
ских методов.

На рисунке 1 представлен обобщенный алгоритм комплексного анализа рисков, включающий в себя подготовительный этап сбора и обработки информации, проработки вариантов решения, выполнения оценки рисков двумя способами (качественным и количественным).

Использование такого алгоритма позволяет:

- определить вероятные риски;

- определить и описать источники возникновения и факторы, на них влияющие;

- описать вероятный ущерб от реализации рисков;

- дать стоимостную оценку вероятному ущербу;

- предложить меры по снижению этих последствий.

При выявлении рисков для определения вероятности наступления события используются объективный и субъективный методы.

Объективных метод предполагает определение вероятности путем вычисления частоты проявления ситуации.

Субъективный метод строится сугубо на ин- дивидуальных данных, основанных на различных предположениях.

Подходы к анализу риска представлены на рисунке 2.

Качественная оценка позволяет произвести идентификацию риска еще на стадии планирования деятельности, определить факторы риска, а также выявить всю цепочку событий, при которой возникает риск.

На данном этапе также проводится стоимостная оценка последствий риска, а также проработка мероприятий по их преодолению.

К количественным методам относятся инструменты теории вероятности и математической статистики. Количественная оценка дает числовое измерение риска, выявляет причины и источники риска, а также позволяет количественно оценить величину вероятных последствий и влияние рисков на эффективность процесса или проекта. Важно отметить, что количественная оценка проводится на базе проведенного качественного анализа [4, с.71].

На рисунке 3 представлена классификация количественных методов оценки риска.

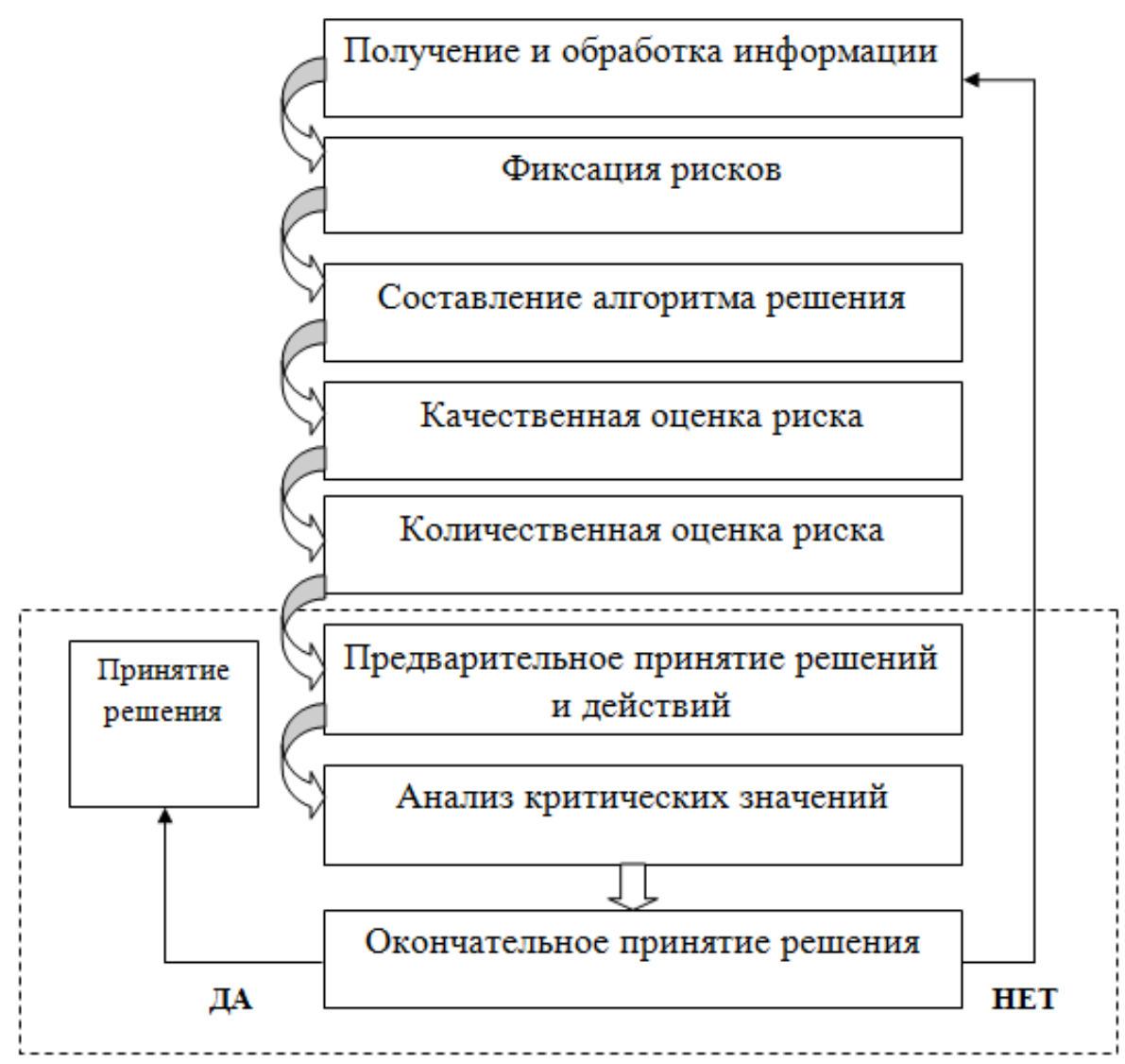

Рисунок 1. Алгоритм комплексного анализа и оценки рисков [6, с.53] 


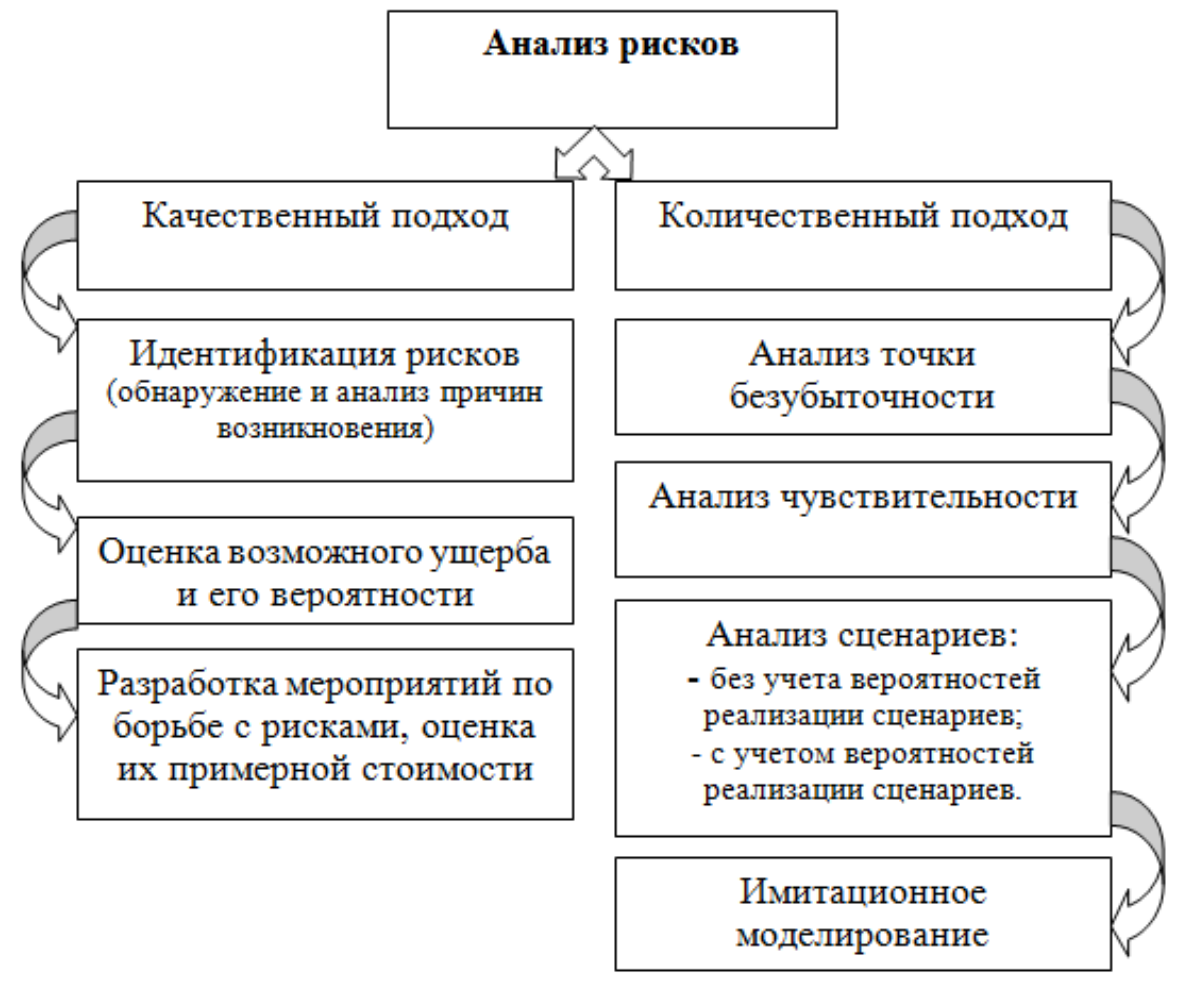

Рисунок 2. Подходы анализа риска [1, с.127]

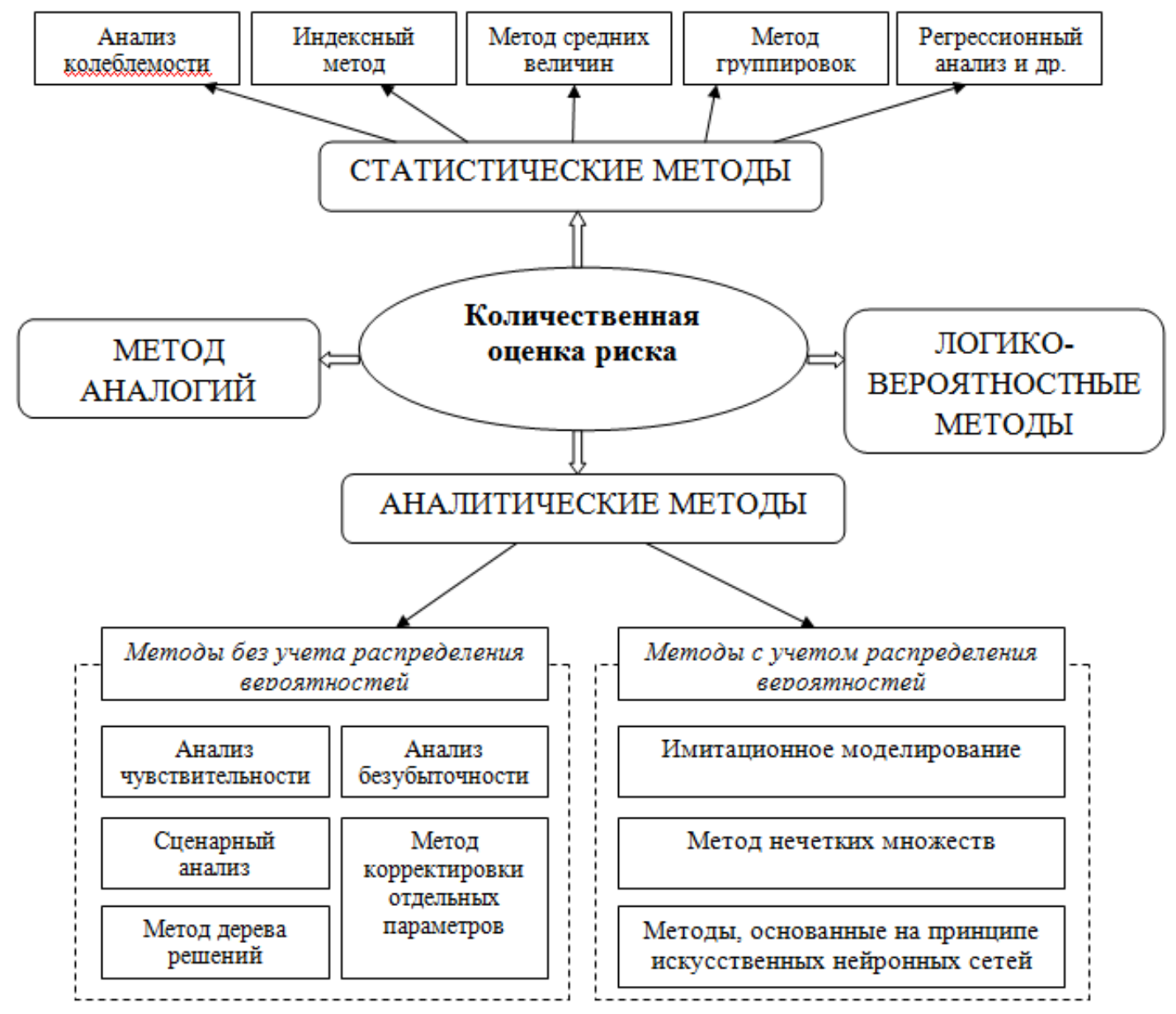

Рисунок 3. Количественные методы оценки рисков 
Наиболее популярными методами являются статистические, т.к. они достаточно просты в плане расчетов и аналитические методы, которые особенно часто используются при оценке рисков в инновационных и инвестиционных проектах.

Описанные выше алгоритмы и методы анализа и оценки рисков могут быть использованы на любом этапе деятельности компании, как в процессе планирования, так и при непосредственной реализации своих задач для корректировки плана действий при случае внезапного проявления рисков.

Только на основании своевременной и достоверной информации можно сформировать адекватное представление о рисках, причинах их возникновения, вероятности наступления и возможных ущербах с оценкой их стоимостного выражения.

Рассмотрим применение количественной оценки риска производства хлебобулочной продукции пекарни «Х». Сегодня пекарни являются стабильно высокорентабельным бизнесом. Средний показатель составляет 19\%. Однако, есть большое количество предприятий, на которых эти показатели доходят до 29-33\%.

Пекарня «Х» в текущем году планирует увеличение производства самых рентабельных видов хлебобулочных изделий, а именно: круассанов, мафинов и донатсов.

Проведем количественный анализ риска пекарни в условиях неопределенности на основе игровой модели по критериям максимажа, минимакса, максимина и критерия пессимизма оптимизма Гурвица.

Прогнозируемые объемы продажи хлебобулочных изделий с учетом текущего спроса следующие:

- ежемесячный объем продаж круассанов с устойчивым спросом в среднем составляет $\mathrm{V}_{1}=$ 330 тыс. руб.;

- ежемесячный объем продаж донатсов с нестабильно устойчивым спросом в среднем составляет $\mathrm{V}_{2}=80$ тыс. руб.;

- ежемесячный объем продаж мафинов обеспечен только разовыми покупками в среднем составляет $\mathrm{V}_{3}=450$ тыс. руб.;

- ежемесячный объем продаж хлебобулочных изделий, спрос на которые не определен, составляет $\mathrm{V}_{4}=130$ тыс. руб.

Проведем расчет прогнозных объемов реализации хлебобулочных изделий.

$$
\mathrm{V}_{1}+\mathrm{V}_{2}+\mathrm{V}_{3}+\mathrm{V}_{4}=330+80+450+130=990 \text { тыс. }
$$
руб.

В соответствии с полученными прогнозными объемами реализации определим три стратегии производства в продажных ценах:

$$
\begin{aligned}
& \mathrm{S}_{1}=\mathrm{V}_{1}+\mathrm{V}_{2}=410 \text { тыс. руб. } \\
& \mathrm{S}_{2}=\mathrm{V}_{1}+\mathrm{V}_{2}+\mathrm{V}_{3}=860 \text { тыс. руб. } \\
& \mathrm{S}_{3}=\mathrm{V}_{1}+\mathrm{V}_{2}+\mathrm{V}_{3}+\mathrm{V} 4=990 \text { тыс. руб. }
\end{aligned}
$$

Далее необходимо рассчитать прибыль пекарни по данным о производстве и прогнозируемых продажах для каждой стратегии с помощью формулы 1. [2]

$$
\left.\mathrm{P}=\mathrm{S}-\mathrm{S}^{\mathrm{c}}-\mathrm{S}^{*} \mathrm{Y}_{\mathrm{vc}}-\mathrm{FC} \text { (при } \mathrm{S} \leqslant \mathrm{V}\right) \text {, }
$$

где S - прогнозируемый объем производства в продажных ценах;

$\mathrm{S}^{\mathrm{C}}$ - себестоимость прогнозируемого объема производства;

$\mathrm{Y}_{\mathrm{vc}}-$ уровень переменных затрат в отношении к объему продаж;

FC - сумма постоянных затрат;

$\mathrm{V}$ - прогнозируемый объем продаж.

Стратегии производства и прогнозируемые значения дохода пекарни «Х» представлены в таблице 1.

Таблица 1. Стратегии производства и прогнозируемые значения дохода пекарни «Х», руб.

\begin{tabular}{|c|c|c|c|c|}
\hline \multirow{2}{*}{ Объем производства } & \multicolumn{5}{|l|}{ Размер дохода в зависимости от прогнозируемых колебаний спроса на хлебобулочные } \\
& $\mathbf{3 3 0 0 0 0}$ & $\mathbf{4 1 0 0 0 0}$ & $\mathbf{8 6 0 0 0 0}$ & $\mathbf{9 9 0 0 0 0}$ \\
\cline { 2 - 5 } & 32010 & 82100 & 82100 & 82100 \\
\hline $\mathrm{S}_{1}=410000$ & -35 & 60300 & 182800 & 182800 \\
\hline $\mathrm{S}_{2}=860000$ & -730 & 39207 & 113437 & 214600 \\
\hline$S_{3}=990000$ & 32010 & 82100 & 182800 & 214600 \\
\hline$M_{j}=\max P_{i j}$ & & & & \\
\hline
\end{tabular}


Критерий максимажа. Наилучшим здесь признается решение, при котором достигается максимальный выигрыш [3].

$$
M=\max \max P_{i j}, 1 \leqslant i \leqslant m ; 1 \leqslant j \leqslant n
$$

$\mathrm{S}_{1}=\max (32010 ; 82100 ; 82100 ; 82100)=$ 82100 руб.

$\mathrm{S}_{2}=\max (-35 ; 60300 ; 182800 ; 182800)=$ 182800 руб.

$\mathrm{S}_{3}=\max (-730 ; 39207 ; 113437 ; 214600)=$ 214600 руб.

$M=\max (82100 ; 182800 ; 214600)=214600$ руб. $\left(\mathrm{S}_{1}\right)$.

Стратегия $\mathrm{S}_{3}$ называется стратегией максимакса, т.е. при любом из условий конъюнктуры рынка лучшим результатом будет $\mathrm{M}=214600$ руб.

Максиминный критерий Вальда. Это позиция крайнего пессимизма [3].

$$
\mathrm{W}=\max \min P_{i j}, 1 \leqslant \mathrm{i} \leqslant \mathrm{m} ; 1 \leqslant \mathrm{j} \leqslant \mathrm{n}
$$

$S_{1}=\min (32010 ; 82100 ; 82100 ; 82100)=32010$ руб.

$S_{2}=\min (-35 ; 60300 ; 182800 ; 182800)=-35$ руб.

$S_{3}=\min (-730 ; 39207 ; 113437 ; 214600)=-730$ руб.

$\mathrm{W}=\max (32010 ;-35 ;-730)=32010$ руб. $\left(\mathrm{S}_{1}\right)$.

Стратегия $S_{1}$ называется максиминной, т.е. при любом из условий конъюнктуры рынка результат будет не хуже, чем $\mathrm{W}=32010$ руб.

Критерий минимаксимума (критерий Сэвиджа). Выбор стратегии аналогичен выбору стратегии по критерию Вальда с тем отличием, что игрок здесь руководствуется матрицей рисков $\mathrm{r}$.
Строим матрицу риска (таблица 2).

Согласно этому критерию стратегия выбирается в соответствии со значением:

$$
S=\min _{1 \leq i \leq m} \max _{1 \leq j \leq n} r_{i j}
$$

где $\mathrm{r}_{\mathrm{ij}}$ - показатель риска.

$\mathrm{r}_{\mathrm{ij}}=\mathrm{M}_{\mathrm{j}}-\mathrm{P}_{\mathrm{j}}$

$\mathrm{M}_{\mathrm{j}}=\max \mathrm{P}_{\mathrm{ij}}$

Таким образом, в соответствии с критерием Сэвиджа следует изготавливать хлебобулочные изделия на сумму 860000 руб.

Критерий пессимизма - оптимизма Гурвица. Согласно этому критерию стратегия выбирается в соответствии со значением:

$\mathrm{H}_{\mathrm{Pi}}=\max \left[\mathrm{p} \min \mathrm{P}_{\mathrm{ij}}+(1-\mathrm{p}) \max \mathrm{P}_{\mathrm{ij}}\right]$ (8).

При этом используется показатель пессимизма - оптимизма «р», который принимает значение $0 \leqslant p \leqslant 1$, и определяется экспертным путем на основании учета различных качественных факторов характеризующих взаимодействие с природой (окружающей средой).

Принимая во внимание современные условия хозяйствования и фактор неопределенности, примем $\mathrm{p}=0,6$.Тогда для каждой стратегии соответственно:

$$
\begin{aligned}
& \mathrm{H}_{\mathrm{P} 1}=0,6 * 32010+(1-0,6) * 82100=52046 \text { руб. } \\
& \mathrm{H}_{\mathrm{P} 2}=0,6 *(-35)+(1-0,6) * 182800=73141 \text { руб. } \\
& \mathrm{H}_{\mathrm{P} 3}=0,6 *(-730)+(1-0,6) * 214600=85402
\end{aligned}
$$
руб.

Выбирается та стратегия, для которой величина $\mathrm{H}_{\mathrm{P}}$ окажется наибольшей, $\mathrm{H}_{\mathrm{Pi}}=\max =85402$ руб, т.е. $S_{3}$.

Результаты выбора стратегий с использованием игровой модели приведены в таблице 3.

Проведенный количественный анализ ри-

Таблица 2. Матрица риска при различных соотношениях прогнозируемого спроса и стратегии производства, руб.

\begin{tabular}{|c|c|c|c|c|c|c|c|}
\hline $\mathbf{r}_{\mathbf{i j}}$ & $\mathbf{3 3 0 0 0 0}$ & $\mathbf{4 1 0 0 0 0}$ & $\mathbf{8 6 0 0 0 0}$ & $\mathbf{9 9 0 0 0 0}$ & $\mathbf{m a x} \mathbf{r}_{\mathbf{j}} \mathbf{m}$ & $\mathbf{m i n m a x} \mathbf{r}_{\mathbf{i j}}$ & $\mathbf{S}_{\mathbf{O \Pi T}}$ \\
\hline $\mathrm{S}_{1}$ & 0 & 0 & 100700 & 132500 & 132500 & - & - \\
\hline $\mathrm{S}_{2}$ & 32045 & 21800 & 0 & 31800 & 32045 & 32045 & 860000 \\
\hline $\mathrm{S}_{3}$ & 32740 & 42893 & 69363 & 0 & 69363 & - & - \\
\hline
\end{tabular}

Таблица 3. Стратегии производства хлебобулочных изделий с учетом показателей затрат, риска и неопределенности по критериям игровой модели, руб.

\begin{tabular}{|c|c|}
\hline Критерии & Планируемый объем производства \\
\hline Критерий максимажа $M$ & 990000 \\
\hline Критерий Вальда $W$ & 410000 \\
\hline Критерий Сэвиджа $r_{i j}$ & 860000 \\
\hline Критерий Гурвица $H_{P i}$ & 990000 \\
\hline
\end{tabular}


сков пекарни «Х» в условиях неопределенности при планируемом увеличении производства хлебобулочных изделий позволяет отметить, что наиболее эффективным является увеличение таких видов хлебобулочных изделий, как круассаны в количестве 3300 шт., на сумму 330000 руб. и донатсы в количестве 1600 шт. на сумму

\section{0 руб.}

Таким образом, применяя количественные методы оценки риска в условиях неопределенности (в частности теорию игр), предприятия смогут гарантированно получать прибыли от производства и реализации наиболее конкурентоспособных видов продукции.

\section{Библиографический список}

1. Анализ эффективности и рисков предпринимательской деятельности: методологические аспекты: монография / Г. В. Савицкая. - 2-е изд., перераб. и доп.-М.: ИНФРА-М, 2019.-291 с.

2. Головина T.A. Методика оценки затрат и показателей риска в условиях выбора эффективного варианта производственной деятельности предприятия / Экономический анализ: теория и практика. - 2010. - № 34 (199).- С. 40-47.

3. Дубов А. М., Лагоша Б. А., Хрусталев Е. Ю. Моделирование рисковых ситуации в экономике и бизнесе: Учебное пособие - М.: Финансы и статистика, 1999.-176 с.

4. Машков Д. М. Совершенствование механизма управления рисками промышленного предприятия: дис. на соиск. уч. степ. канд. экон. наук.-М., 2015.

5. Райзберг Б.А., ЛозовскийЛ.Ш., Стародубцева Е.Б.. Современный экономический словарь.- 2-е изд., испр. М.: ИНФРА-М. 479 с.. 1999.

6. Серов С.Н. Методические особенности оценки инвестиционных процессов в условиях финансовой неустойчивости предприятий: дис. на соиск. уч. степ. канд. экон. наук. - Ставрополь, 2005. 